\title{
Organic complementary-like inverters employing methanofullerene-based ambipolar field-effect transistors
}

\author{
Thomas D. Anthopoulos, ${ }^{\text {a) }}$ Dago M. de Leeuw, Eugenio Cantatore, \\ Sepas Setayesh, and Eduard J. Meijer \\ Philips Research Laboratories, Prof. Holstlaan 4 (WAG), 5656 AA Eindhoven, The Netherlands \\ Cristina Tanase, Jan C. Hummelen, and Paul W. M. Blom \\ Molecular Electronics, Materials Science Centre Plus, University of Groningen, Nijenborgh 49747 AG \\ Groningen, The Netherlands
}

(Received 9 July 2004; accepted 13 September 2004)

\begin{abstract}
We demonstrate a complementary-like inverter comprised of two identical ambipolar field-effect transistors based on the solution processable methanofullerene [6,6]-phenyl- $\mathrm{C}_{61}$-butyric acid methyl ester (PCBM). The transistors are capable of operating in both the $p$ - and $n$-channel regimes depending upon the bias conditions. However, in the $p$-channel regime transistor operation is severely contact limited. We attribute this to the presence of a large injection barrier for holes at the $\mathrm{Au} / \mathrm{PCBM}$ interface. Despite this barrier the inverter operates in both the first and third quadrant of the voltage output versus voltage input plot exhibiting a maximum gain in the order of 20. Since the inverter represents the basic building block of most logic circuits we anticipate that other complementary-like circuits can be realized by this approach. () 2004 American Institute of Physics.

[DOI: $10.1063 / 1.1812577]$
\end{abstract}

Organic field-effect transistors (OFETs) are currently the focus of intense research efforts in numerous academic and industrial research laboratories around the world. Over the past decade OFETs have emerged as promising candidates for electronic device applications requiring low cost and large area coverage, mechanical flexibility, and low temperature processing. ${ }^{1}$ Several groups have demonstrated OFETbased circuits with performances sufficient for practical applications. Few examples of such applications include switching devices for flat panel displays ${ }^{2-4}$ and integrated circuits. ${ }^{4-6}$ To date the largest organics-based electronic circuit reported is the standard logic based 32-stage shift registers that consists of 1888 transistors capable of operating at a frequency of $5 \mathrm{kHz}{ }^{4}$ As the number of transistors per circuit increases there is an increasing need for circuits characterized by low power dissipation, high noise margin, and greater operation stability. In silicon-based microelectronics such requirements are met through the use of complementary metal-oxide-semiconductor (CMOS) logic where an $n$ - and a $p$-type transistor are combined to built logic circuits. Indeed, examples of organic CMOS logic have show that it is possible to make large-scale integrated circuits (up to 864 transistors per circuit) with much lower power dissipation. ${ }^{6}$ In the work by Crone et al., however, the transistor channels had to be spatially separated in order to facilitate the separate vacuum deposition of the two semiconductors (an $n$ - and a $p$-type), thus making circuit fabrication difficult and potentially expensive.

Recently an alternative approach towards organic CMOS circuits has been proposed. ${ }^{7}$ In their work Meijer et al. have demonstrated that by employing identical ambipolar OFETs based on polymer-small molecule interpenetrating networks as well as narrow band gap polymers, CMOS-like voltage inverters can be fabricated. This approach makes full use of

\footnotetext{
a) Author to whom correspondence should be addressed; electronic mail:
} thomas.anthopoulos@philips.com the attractive processing properties of polymers while it simplifies device fabrication by utilizing a single semiconductor layer. However, despite these promising preliminary results there is still a drawback associated with most ambipolar OFETs reported to date, which is the relatively low carrier mobility. ${ }^{7-10}$ For example, in the work by Meijer et al. the maximum ambipolar carrier mobilities reported were in the order of $10^{-5} \mathrm{~cm}^{2} / \mathrm{V} \mathrm{s}$ (for both electrons and holes) for narrow band gap based polymeric OFETs, and $10^{-5} \mathrm{~cm}^{2} / \mathrm{V} \mathrm{s}$ (electrons) $10^{-3} \mathrm{~cm}^{2} / \mathrm{V} \mathrm{s}$ (holes) for OFETs based on polymer-small molecule interpenetrating networks. ${ }^{7}$ Moreover, Dodabalapur et al. have observed that the electron mobility in ambipolar OFETs employing an organic heterostructure of $\mathrm{C}_{60} / \alpha-6 \mathrm{~T}$ is lower by a factor of 16 when compared with the electron mobility measured in pristine $\mathrm{C}_{60}$ OFETs. ${ }^{8,11}$ Since carrier mobility is the main limiting factor in the operating frequency of complementary organic circuits, ideally, one would like to have materials with much higher mobilities for both electrons and holes. ${ }^{12,13}$

We have recently discovered that OFETs based on the solution processable methanofullerene derivative [6,6]phenyl- $\mathrm{C}_{61}$-butyric acid methyl ester (PCBM) exhibit ambipolar transport characteristics even when a high work function metal $(\mathrm{Au})$ is employed as the source and drain electrodes. ${ }^{14}$ We found that the carrier mobilities in this particular methanofullerene are in the order of 1 $\times 10^{-2} \mathrm{~cm}^{2} / \mathrm{V}$ s for electrons and $8 \times 10^{-3} \mathrm{~cm}^{2} / \mathrm{V}$ s for holes with on-off current ratios $\sim 10^{6}$. Here, we demonstrate that PCBM based ambipolar OFETs can be used for fabrication of CMOS-like logic inverters with good operating characteristics.

Field-effect transistors were made using heavily doped $p$-type $\mathrm{Si}$ wafers as the common gate electrode with a $200 \mathrm{~nm}$ thermally oxidized $\mathrm{SiO}_{2}$ layer as the gate dielectric. Using conventional photolithography, gold source and drain electrodes were defined in a bottom contact configuration with channel width of $1000 \mu \mathrm{m}$ and lengths in the range 

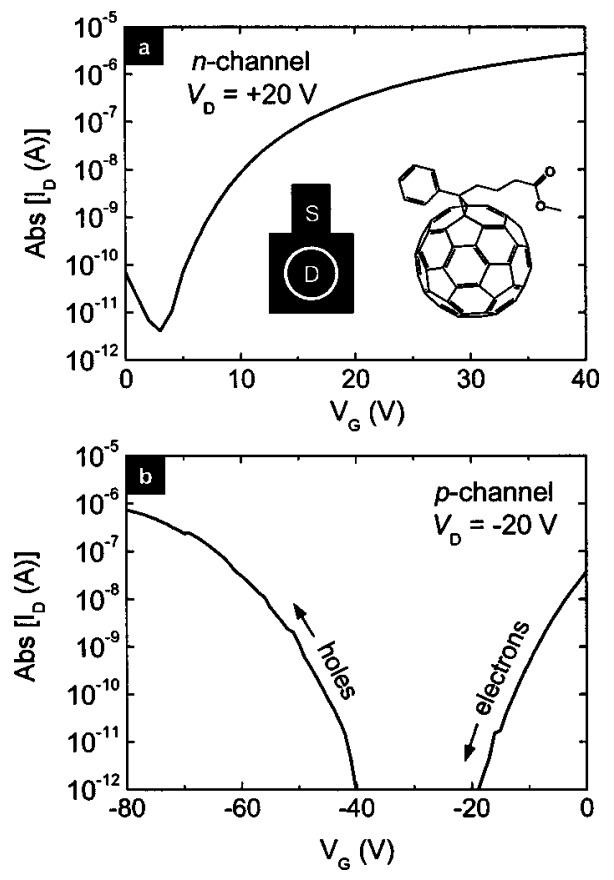

FIG. 1. Room temperature transfer characteristics for a PCBM based OFET with a characteristic channel length and width of 20 and $1000 \mu \mathrm{m}$, respectively. (a) Transistor operation in electron accumulation at $V_{D}=+20 \mathrm{~V}$. Inset shows the schematic diagram of the top view of the ring-type transistor geometry used and the molecular structure of PCBM. (b) Transistor operation in hole accumulation at $V_{D}=-20 \mathrm{~V}$. The drain current in (b) observed for $V_{G}$ in the range $\left|V_{D}\right|>\left|V_{G}\right| \geqslant 0 \mathrm{~V}$ is due to electron contribution (see Refs. 7, 10, and 14).

0.75-40 $\mu \mathrm{m}$. A $10 \mathrm{~nm}$ layer of titanium was used acting as an adhesion layer for the gold on $\mathrm{SiO}_{2}$. The $\mathrm{SiO}_{2}$ layer was treated with the primer hexamethyldisilazane prior to semiconductor deposition in order to passivate its surface. The drain electrode was contained within a circular source electrode [inset Fig. 1(a)] in order to minimize parasitic leakage currents. ${ }^{15}$ Films were spun from a $10 \mathrm{mg} / \mathrm{ml}$ solution of PCBM in chlorobenzene at $500 \mathrm{rpm}$ for $1 \mathrm{~min}$. The molecular structure of PCBM is shown in inset of Figs. 1(a) and 1(b). All freshly prepared devices were annealed in vacuum of $10^{-7}$ mbar at $120^{\circ} \mathrm{C}$ for several hours. All electrical measurements were performed in high vacuum of $10^{-7}$ mbar at room temperature $\left(24^{\circ} \mathrm{C}\right)$ using an HP $4156 \mathrm{~B}$ semiconductor parameter analyzer.

Figure 1 shows the transfer characteristics of an ambipolar PCBM based OFET $(L=20 \mu \mathrm{m}, W=1000 \mu \mathrm{m})$ in electron enhancement [Fig. 1(a)] and hole enhancement [Fig. 1(b)] operating mode. Maximum electron and hole mobilities measured are $1 \times 10^{-2} \mathrm{~cm}^{2} / \mathrm{V} \mathrm{s}$ (at $V_{G}=20 \mathrm{~V}, V_{D}=20 \mathrm{~V}$ ), and $8 \times 10^{-3} \mathrm{~cm}^{2} / \mathrm{V} \mathrm{s} \quad\left(\right.$ at $\quad V_{G}=-75 \mathrm{~V}, \quad V_{D}=-20 \mathrm{~V}$ ), respectively. ${ }^{14}$ From Fig. 1(b) it is evident that for $p$-channel operation the transistor exhibit a high switch-on voltage of approximately $-40 \mathrm{~V}$. This observation suggests either the presence of a significant density of hole traps at the $\mathrm{SiO}_{2} / \mathrm{PCBM}$ interface and/or the existence of a large contact barrier for hole injection from $\mathrm{Au}$ into the higher occupied molecular orbital of PCBM. ${ }^{14}$ The barrier to electron injection from $\mathrm{Au}$ into the lower unoccupied molecular orbital (LUMO) of PCBM, on the other hand, appears to be relatively small exhibiting a switch-on voltage of only $+3 \mathrm{~V}$ [see Fig. 1(a)]. were identical and equal to $L=10 \mu \mathrm{m}, W=1000 \mu \mathrm{m}$. In the
Downloaded 25 Apr 2008 to 155.198.4.89. Redistribution subject to AIP license or copyright; see http://apl.aip.org/apl/copyright.jsp
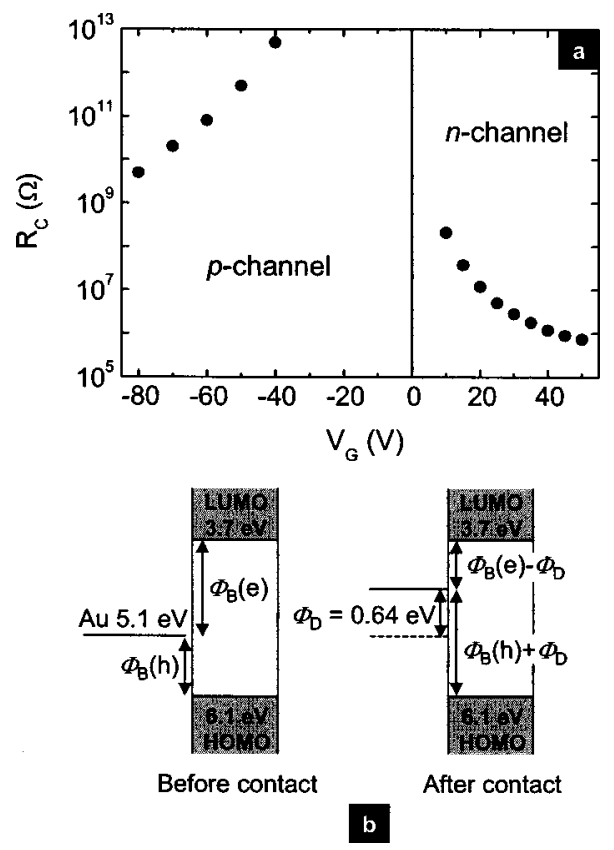

FIG. 2. (a) Extracted contact resistance $\left(R_{C}\right)$ vs gate bias $\left(V_{G}\right)$ for PCBM based OFETs. The value of $R_{C}$ was calculated in the linear regime $\left(\left|V_{D}\right|\right.$ $=2 \mathrm{~V}$ ) in both $p$ - and $n$-channel mode of operation. (b) Simplified energy level diagram for Au and PCBM before and after contact.

In order to obtain some insight in the contact effects present in our devices we have calculated the contact resistance $R_{C}$ (drain + source contacts) using the transmission line method described elsewhere. ${ }^{16-18}$ Figure 2(a) shows the derived values of $R_{C}$ as a function of gate voltage in $n$ - as well as $p$-channel operating regime. It is evident that for both channels $R_{C}$ is strongly dependent on $V_{G}$. The contact resistance for the $p$ channel, however, is several orders of magnitude higher than the values measured for the $n$ channel, implying a severely contact limited device operation. Such high resistance is consistent with the presence of a rather large injection barrier for holes ${ }^{14}$ and can be better understood in terms of the energy level diagram shown in Fig. 2(b). In this diagram the energy difference between the Fermi level of $\mathrm{Au}$ and the energy levels of PCBM, before and after contact, are shown. For simplicity, here the energy levels are drawn flat but in reality band bending occurs upon contact as well as upon application of a gate bias. From the band offsets (before contact), potential barriers $\Phi_{B}(h)$ $\sim 1 \mathrm{eV}$ for holes and $\Phi_{B}(e) \sim 1.4 \mathrm{eV}$ for electrons, are expected. Contrary to our expectations, however, such barrier heights are in contrast with the experimental data of Fig. 1. We ascribe this discrepancy to a shift in the vacuum potential at the interface $\left(\Phi_{D}\right)$ towards the LUMO level of PCBM by $\sim 0.64 \mathrm{eV}$, upon contact. ${ }^{19}$ Taking this shift into account the injection barrier for electrons is expected to decrease $\left[\Phi_{B}(e)-\Phi_{D}=0.76 \mathrm{eV}\right]$, while the barrier for holes to increase by an equal amount $\left[\Phi_{B}(h)+\Phi_{D}=1.64 \mathrm{eV}\right]$. Although model of Fig. 2(b), they are qualitatively consistent with the experimental data of Figs. 1 and 2(a).

The transfer characteristics of a PCBM-based complementary-like inverter are shown in Fig. 3. The inset in Fig. 3(a) displays the schematic of the CMOS-like inverter circuit employed. The channel dimensions for both OFETs these predictions are based on the simplified energy level 

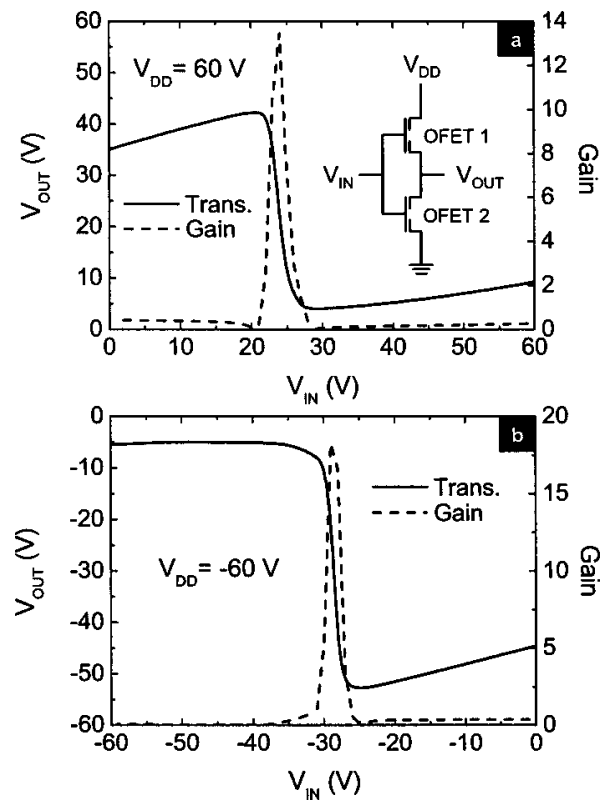

FIG. 3. Transfer characteristics of a CMOS-like inverter comprised of two identical PCBM-based ambipolar OFETs $(L=10 \mu \mathrm{m}, W=1000 \mu \mathrm{m})$. (a) Inverter characteristics (solid line) with $V_{\mathrm{IN}}$ and $V_{\mathrm{DD}}$ being positively biased and the corresponding gain (dashed line). Inset shows the inverter circuit configuration. (b) Inverter characteristics with $V_{\mathrm{IN}}$ and $V_{\mathrm{DD}}$ being negatively biased.

circuit the gate is common for both transistors and serves as the input node $\left(V_{\mathrm{IN}}\right)$. When the supply voltage $\left(V_{\mathrm{DD}}\right)$ and $V_{\mathrm{IN}}$ are biased positively [Fig. 3(a)] the inverter works in the first quadrant of the output voltage $\left(V_{\text {OUT }}\right)$ vs $V_{\text {IN }}$ plot exhibiting a maximum gain of 13. Under these bias conditions OFET 1 acts mainly like the $p$-type transistor of a regular CMOS inverter while OFET 2 operates as the $n$-type device. If the input and the bias voltage are negative, the inverter still operates (gain $\sim 18$ ) with $n$ - and $p$-channel function exchanged between the two devices. With reference to Fig. 3(a), when $V_{\mathrm{IN}}$ is close to $V_{\mathrm{DD}}$, the $n$-channel of both transistors is on with OFET 1 having a smaller overdrive than OFET 2. This explains why the output node cannot be completely pulled down by OFET 2 and why the output voltage slightly decreases with decreasing $V_{\mathrm{IN}}$. When $V_{\mathrm{IN}}$ is $\sim 40 \mathrm{~V}$ lower than $V_{\text {DD }}$ the $p$-channel of OFET 1 and OFET 2 are on with a smaller overdrive on OFET 2. Again this explains the incomplete pull-up and the slight reduction of the output voltage with $V_{\mathrm{IN}}$. Similar considerations apply to Fig. 3(b). It is worth noting that the ability to operate in both quadrants of the $V_{\text {OUT }}$ VS $V_{\text {IN }}$ plot is a unique feature of ambipolar inverters since unipolar ones operate only in one quadrant (i.e., either in the first or third).
In summary, we have demonstrated a complementarylike voltage inverter comprised of two identical ambipolar OFETs based on the solution processable methanofullerene PCBM. The inverter can function at room temperature exhibiting a maximum voltage gain of 18 . This is one of the highest gains reported to date for OFET based inverters. Furthermore, the use of high mobility ambipolar organic semiconductors such as PCBM can be viewed as a significant step towards organic-based CMOS-like technology.

T.D.A. gratefully acknowledges financial support from the EC under Project No. HPRN-CT-2002-00327 (EUROFET). The work of C.T. forms part of the research programme of the Dutch Polymer Institute Project No. 276.

${ }^{1}$ D. Voss, Nature (London) 407, 442 (2000).

${ }^{2}$ N. D. Young, R. M. Bunn, R. W. Wilks, D. J. McCulloch, S. C. Deane, M. J. Edwards, G. Harkin, and A. Pearson, J. Soc. Inf. Disp. 5/3, 275 (1997).

${ }^{3}$ H. Sirringhaus, N. Tessler, and R. H. Friend, Science 280, 1741 (1998).

${ }^{4}$ G. H. Gelinck, H. E. A. Huitema, E. van Veenendaal, E. Cantatore, L. Schrijnemakers, J. B. P. H. van der Putten, T. C. T. Geuns, M. Beenhakkers, J. B. Giesbers, B.-H. Huisman, E. J. Meijer, E. M. Benito, F. J. Touwslager, A. W. Marsman, B. J. E. van Rens, and D. M. de Leeuw, Nat. Mater. 3, 106 (2004).

${ }^{5}$ C. J. Drury, C. M. Mutsaers, C. M. Hart, M. Matters, and D. M. de Leeuw, Appl. Phys. Lett. 73, 108 (1998).

${ }^{6}$ B. Crone, A. Dodabalapur, Y.-Y. Lin, R. W. Fillas, Z. Bao, A. LaDuca, R. Sarpeshkar, H. E. Katz, and W. Li, Nature (London) 403, 521 (2000).

${ }^{7}$ E. J. Meijer, D. M. de Leeuw, S. Setayesh, E. Van Veenendaal, B.-H. Huisman, P. W. M. Blom, J. C. Hummelen, U. Scherf, and T. M. Klapwijk, Nat. Mater. 2, 678 (2003).

${ }^{8}$ A. Dodabalapur, H. E. Katz, L. Torsi, and R. C. Haddon, Appl. Phys. Lett. 68, 1108 (1996).

${ }^{9}$ K. Tada, H. Harada, and K. Yoshino, Jpn. J. Appl. Phys., Part 2 35, L944 (1996).

${ }^{10}$ R. J. Chesterfield, C. R. Newman, T. M. Pappenfus, P. C. Ewbank, M. H. Haukaas, K. R. Mann, L. L. Miller, and C. D. Frisbie, Adv. Mater. (Weinheim, Ger.) 15, 1278 (2003).

${ }^{11}$ R. C. Haddon, A. S. Perel, R. C. Morris, T. T. M. Palstra, A. F. Hebard, and R. M. Fleming, Appl. Phys. Lett. 67, 121 (1995).

${ }^{12}$ A. R. Brown, A. Pomp, C. M. Hart, and D. M. de Leeuw, Science 270, 972 (1995).

${ }^{13}$ C. D. Dimitrakopoulos and P. R. L. Malenfant, Adv. Mater. (Weinheim, Ger.) 14, 99 (2002).

${ }^{14}$ T. D. Anthopoulos, C. Tanase, S. Setayesh, E. J. Meijer, J. C. Hummelen, P. W. M. Blom, and D. M. de Leeuw, Adv. Mater. (Weinheim, Ger.) (to be published)

${ }^{15}$ E. J. Meijer, C. Detcheverry, P. J. Baesjou, E. van Veenendaal, D. M. de Leeuw, and T. M. Klapwijk, J. Appl. Phys. 93, 4831 (2003).

${ }^{16}$ J. Zaumseil, K. W. Baldwin, and J. A. Rogers, Appl. Phys. Lett. 93, 6117 (2003).

${ }^{17}$ E. J. Meijer, G. H. Gelinck, E. Van Veenendaal, B.-H. Huisman, D. M. de Leeuw, and T. M. Klapwijk, Appl. Phys. Lett. 82, 4576 (2003).

${ }^{18}$ B. H. Hamadani and D. Natelson, Appl. Phys. Lett. 84, 443 (2004).

${ }^{19}$ J. K. J. van Duren, V. D. Mihailetchi, P. W. M. Blom, T. van Woudenbergh, J. C. Hummelen, M. T. Rispens, R. A. J. Janssen, and M. M. Wienk, J. Appl. Phys. 94, 4477 (2003). 